\title{
Second International Conference on Evidence-based Dentistry
}

\author{
Ben Balevi \\ Dental Practitioner, Vancouver, British Columbia, Canada
}

Evidence-Based Dentistry (2006) 7, 3. doi:10.1038/sj.ebd.6400376

One hundred and thirty-two delegates met in Chicago, USA on November 5 and 6, 2006 to attend the Second International Conference on Evidence-based Dentistry. A preconference workshop was held on 4 November, led by Jane Forrest (University of California-Los Angeles), Philip Hujoel (University of Washington) and Pamela Overman (University of Missouri) who reviewed the basic principles of evidence-based practice. Subjects included good question formulation, searching for evidence, critically appraising the evidence and how to implement the evidence in practice.

The conference was officially opened the next morning by the conference chairperson, Philip Hujoel, introducing the concept of the 'precautionary principle'. This principle promotes taking precautionary measures against any activity that may raise threats or harm to humans or the environment, if there is a perceived causality, even in the absence of scientific evidence. The approach was defended by Joel Tickner (University of Massachusetts-Lowell), whereas Noel Weiss (University of Washington) suggested that, "...caution be taken when subscribing to the precautionary principle". He presented evidence of how the precautionary principle may obstruct the advancement of valid health-science outcomes. Susan Runner (US Food and Drug Administration) concluded the morning's plenary sessions by reviewing the Administration's approval process of medical devices.

Two concurrent sessions ran in the afternoon. John Gunsolley moderated the session, "Are we practising according to evidence?" Paul Robertson (University of Washington) and Han Berston
(University of Sweden) reviewed the current evidence on the risk of smoking on the periodontium, and on the diagnosis, prognosis and treatment outcomes of periodontitis. Jim Fricton (University of Minnesota) and Christian Stohler reviewed the current evidence on the diagnosis, prognosis and available treatment options for temporomandibular disorder.

The parallel afternoon session was moderated by Jane Forrest and Debora Mathews (Dalhousie University). Speakers included Richard Stevenson (University of California-Los Angeles), Ralph Katz (New York University College of Dentistry) and Dr Forrest herself, reviewing the evidence on successfully integrating evidence-based principles into the education system. Gordon Douglass (Sacramento, CA), David Turpin (University of Washington) and Barbara Gooch (US Centers for Disease Control) discussed the integration of evidence-based practice into setting clinical guidelines.

The next day began again with the concurrent sessions, "Pushing the boundaries of dental treatment" and "Real world implementation of evidence-based dental practice", moderated by James Bader (University of North Carolina) and Mar Cruz (University of California, Los Angles), respectively. David Cochran (University of Texas) presented a review of the clinical success of immediate and early loading of dental implants, followed by Dr Bader describing classical evidence-based decisionanalysis on the diagnosis and treatment of incipient or suspected occlusal caries in adults. Shane White (University of California-Los Angeles) concluded the session by trying to answer the clinical question of when to save a tooth with endodontic therapy in lieu of sacrificing it for the sake of a single tooth implant replacement.

Later in the day, talks were given by Daniel Myer (University of Virginia), Maxwell Anderson (University of Washington) and Dave Famili (Santa Monica Dental Group, CA), who discussed evidence-based dentistry (EBD) from the perspectives, respectively, of the American Dental Association (ADA), third-party payers and peer-review committees. Mark Cruz showed how EBD has been implemented into the "real world" and Richard Koa reviewed some of the challenges of transferring EBD into clinical practice.

The conference concluded on Sunday afternoon with a plenary session on professional licensure. John Crosby (American Association of Dental Examiners) argued in favour of a single national clinical licensure examination, which can then be defended legally. On the other hand, William Calnon (New York State University-Buffalo) strongly argued in favour of compulsory hospital-based dental residency programmes as a requirement for licensure. Richard Ranney and Howard Jones (ADA) reviewed the evidence on the effectiveness of clinical licensure examinations and the role the ADA is playing in establishing guidelines for them.

Closing remarks were delivered by Dr Hujoel. He commented how this conference was a natural continuation of the first international conference on EBD, held in Atlanta 2 years ago. Hopefully, the continued advancement in EBD movement will encourage a larger gathering at the third conference, to be held in 2007. 